\title{
MONITORAMENTO DE INTEGRIDADE ESTRUTURAL BASEADO EM IMPEDÂNCIA ELETROMECÂNICA UTILIZANDO O MÉTODO DE KRIGAGEM ORDINÁRIA
}

\author{
D.R. GONÇALVES ${ }^{1}$, J.R.V. MOURA JUNIOR ${ }^{2}$, P.E.C. PEREIRA ${ }^{3}$ \\ Universidade Federal de Catalão \\ danielresendeg@gmail.com ${ }^{1}$ \\ Submetido 30/01/2020 - Aceito 16/03/2020 \\ DOI: $10.15628 /$ holos.2020.9480
}

\section{RESUMO}

A ocorrência de falhas estruturais em equipamentos e estruturas civis decorre de diversos fatores, tais como impacto, atrito e fadiga, demandando esforços para sua correção. Entretanto, em virtude de riscos de segurança que tais falhas representam no meio industrial, torna-se necessário o monitoramento das estruturas e equipamentos, a fim de identificar rapidamente o início de uma falha, o que permite a tomada de medidas preventivas em tempo hábil. Diante deste cenário, foi utilizado, neste trabalho, a Krigagem Ordinária para a interpolação de dados numéricos de métrica de dano baseados em impedância eletromecânica (E/M) em uma placa de alumínio. Os resultados mostraram que o método foi capaz de mapear as regiões de falhas, além de evidenciar direções preferenciais de propagação do dano estrutural. Por meio da variância de krigagem foram mapeadas áreas com deficiência de sensores (PZTs), de tal maneira que tal informação poderia fornecer diretrizes para o estabelecimento de malhas de sensores no sentido de determinar arranjos mais adequados para a coleta de informações da estrutura analisada. A partir dos resultados obtidos, faz-se necessária a realização de pesquisas adicionais para aprimorar a aplicação do método, entender suas limitações e analisar a viabilidade de implantação no meio industrial.

PALAVRAS-CHAVE: previsão de falhas, estimativa, krigagem ordinária, dados numéricos.

\section{STRUCTURAL HEALTH MONITORING BASED ON ELECTROMECHANICAL IMPEDANCE USING THE ORDINARY KRIGING METHOD}

\begin{abstract}
The occurrence of structural failures in equipment and civil structures accrues from several factors, such as impact, friction and fatigue, requiring efforts for their correction. However, due to safety risks that such failures represents in the industrial environment, it is necessary to monitor the structures and equipment with the aim of quickly identify the beginning of a failure, which allows preventive measures to be taken in a timely manner. Given this scenario, the Ordinary Kriging was used in this work to interpolate numerical data of damage metric based on electromechanical (E/M) impedance in an aluminum plate. The results shows that the method was

able to map the failure areas, in addition to evidences preferred directions of structural failure propagation. Through kriging variance, were mapped areas with sensor (PZTs) deficiency, in such a way that such information could provide guidelines for the establishment of sensor grids in order to determine more appropriate arrangements for collecting information form the analyzed structure. From the results obtained it is necessary to carry out additional research with the purpose of improve the method application, understand its limitations and analyze the feasibility of implantation in the industrial environment.
\end{abstract}

KEYWORDS: fault prediction, estimate, ordinary kriging, numerical data. 


\section{INTRODUÇÃO}

A ocorrência de falhas em equipamentos industriais e estruturas está associada normalmente a três causas: impacto, atrito e fadiga. A identificação e a reparação das falhas (manutenção) são essenciais para que um equipamento/estrutura funcione de acordo com as especificações de projeto, e requerem um sistema (sensores, equipamentos de aquisição e transferência de dados, análise de dados, modelagem das condições, alarmes, etc) para o monitoramento da estrutura/equipamento (Bao et al., 2019). O mapeamento das condições estruturais depende fundamentalmente da deteç̧ão da falha, o que envolve localizar, quantificar e estimar sua magnitude (Farrar, Lieven, \& Bement, 2005).

A manutenção de estruturas/equipamentos é altamente necessária em função dos altos custos operacionais e de suporte dos sistemas de engenharia (Dhillon, 2008). Ainda, o funcionamento de estruturas próximo ao limite de operação das mesmas demandam políticas de manutenção mais rigorosas. Para o monitoramento de tais estruturas, diversas abordagens foram criadas e têm sido aplicadas, principalmente nas duas últimas décadas (Moura Junior, 2008), dentre as quais tem-se: extensometria, ultrassom, radiografia, correntes parasitas, análise de vibrações tradicional, emissão acústica, vácuo comparativo, ondas de Lamb e impedância eletromecânica (Moura Junior, 2008; Bray \& McBride, 1992).

As manutenções podem ser realizadas por meio de duas abordagens: (i) substituição de peças que se julga comprometidas (manutenção corretiva); e (ii) diagnóstico e correção prévia de problemas identificados, antes da ocorrência da falha estrutural (manutenção preditiva) (Moura Junior, 2008). Diante do fato de que aproximadamente $20 \%$ do aço produzido no mundo é utilizado na reposição de perdas causadas pela corrosão (manutenção corretiva), é imperativo a utilização de um sistema de manutenção capaz de prever a confiabilidade do equipamento/estrutura (manutenção preditiva), o que poderia reduzir os custos advindos de tal operação (Silva, 2017).

Um dos métodos utilizados na manutenção preditiva é o Monitoramento de Integridade Estrutural (Structural Health Monitoring - SHM) baseado em impedância eletromecânica, a qual é classificada como uma técnica de avaliação não destrutiva, pois não danifica a estrutura ao longo de seu monitoramento (Silva, 2017; Baptista, 2010; Farrar, Lieven, \& Bement, 2005). Nessa abordagem, dados são coletados por meio de uma rede integrada de sensores, os quais fornecem informações pontuais sobre a integridade estrutural, necessitando-se de uma interpolação dos valores indicados pelos sensores para o mapeamento de toda a estrutura.

Diante da necessidade de modelagem (interpolação) dos dados de impedância eletromecânica, propõe-se com este trabalho, a partir de dados numéricos semelhantes a valores reais, utilizar um método geoestatístico (Krigagem Ordinária) para interpolar as informações de dano estrutural em uma placa de alumínio, considerando-se que tais informações possuem uma correlação espacial para tal. A partir da interpolação, por Krigagem Ordinária, de valores de métricas de dano baseados em impedância eletromecânica, pode-se mapear condições de falhas em termos de localização e gravidade. 


\section{REVISÃO BIBLIOGRÁFICA}

A partir do tema e das etapas realizadas neste trabalho, estão descritos abaixo os conceitos pertinentes, os quais são: (i) Monitoramento de Integridade Estrutural; e (ii) Teoria Geoestatística, especificamente, Variograma e Krigagem Ordinária.

\subsection{Monitoramento de Integridade Estrutural}

Recorrentemente em estruturas e equipamentos industriais ocorrem falhas, geralmente causadas por fadiga, impacto e/ou atrito. Para um sistema funcionar corretamente, tais falhas devem ser localizadas e reparadas oportunamente, sendo que a deteç̧ão de uma falha é um problema que compreende localizar, quantificar e estimar a vida útil excedente (Farrar, Lieven, \& Bement, 2005).

O Monitoramento de Integridade Estrutural tem o propósito de fornecer ferramentas para um monitoramento constante ou periódico de equipamentos e estruturas, no sentido de avaliar a integridade estrutural e predizer a vida útil dos mesmos, fornecendo embasamento para a execução de ações remediadoras locais, o que reduz o custo geral de manutenção ao otimizar a alocação de recursos (Yan, Chen, \& Mukhopadhyay, 2017; Gopalakrishnan, Ruzzene, \& Hanagud, 2011).

O monitoramento de estruturas baseado em uma rede de sensores piezelétricos está entre as abordagens mais utilizadas, em virtude da facilidade operacional, baixo custo de implantação e ser não invasiva, podendo ser realizada por meio de vários métodos: (i) propagação de ondas; (ii) função de transferência de resposta em frequência; e (iii) impedância eletromecânica (Eletromechanical (E/M) impedance) (Giurgitiu, 2014; Gopalakrishnan, Ruzzene, \& Hanagud, 2011).

$\mathrm{Na}$ técnica baseada em impedância eletromecânica $(E / M)$, um elemento piezelétrico, geralmente um PZT (lead zirconate-titanate), é embutido ou anexado à estrutura por meio de uma cola adesiva de alta resistência e sujeito a uma tensão sinusoidal, de tal forma que o elemento piezelétrico retorna para um medidor a impedância eletromecânica, cujos resultados são plotados em função da frequência, resultando em um mapeamento das condições estruturais iniciais. Uma alteração nos valores de impedância eletromecânica revela a presença de danos na estrutura por meio da comparação entre as assinaturas da impedância inicial e após o dano (Maruo, Giachero, Steffen Junior, \& Finzi Neto, 2015; Bhalla \& Soh, 2012; Zagrai \& Giurgiutiu, 2009).

A expressão em termos de impedância elétrica de um transdutor é dada pela Equação (1) (Liang, Sun, \& Rogers, 1994), na qual $Z_{E}(\omega)$ é a impedância elétrica do transdutor; $Z_{S}(\omega)$, a impedância mecânica da estrutura; $Z_{p}(\omega)$, a impedância mecânica do transdutor, $\omega$, frequência angular; $\tau$, constante geométrica; $\epsilon_{33}^{T}$, constante dielétrica para uma tensão mecânica $(T)$ constante; $Y_{X X}^{L}$, módulo de Young para um campo elétrico $(E)$ constante; $d_{3 X}^{2}$, constante piezelétrica; e $j$, unidade imaginária.

$Z_{E}(\omega)=\frac{1}{j \omega \tau}\left(\epsilon_{33}^{T}-\frac{Z_{s}(\omega)}{Z_{S}(\omega)+Z_{p}(\omega)} d_{3 X}^{2} Y_{X X}^{E}\right)^{-1}$ 
O mapeamento de falhas é realizado por meio de índices de métrica de dano, derivados dos valores de impedância elétrica (Equação 1). Entre esses, o Desvio da Raiz Média Quadrática (Root Mean Square Deviation - RMSD), calculado por meio da Equação (2), é a medida quantitativa mais comumente utilizada para se mensurar a similaridade/correlação entre valores localizados em duas coordenadas atômicas sobrepostas, fundamentada na norma Euclidiana (Giurgiutiu, 2014; Giurgiutiu \& Rogers, 1998).

$R M S D=\sqrt{\frac{\sum_{k=\omega_{I}}^{\omega_{F}}\left[Z_{E}(k)-Z_{E}^{0}(k)\right]^{2}}{\sum_{k=\omega_{I}}^{\omega_{F}}\left[Z_{E}^{0}(k)\right]^{2}}}$

Na Equação (2), $Z_{E}(k)$ e $Z_{E}^{0}(k)$, são, respectivamente, as assinaturas de impedância elétrica posterior e anterior à ocorrência de dano, ambas medidas na frequência $k$, a qual varia de $\omega_{I}$ (frequência inicial) à $\omega_{F}$ (frequência final).

\subsection{Teoria Geoestatística}

A Geoestatística foi desenvolvida na década de 1950 como uma aplicação da Teoria das Variáveis Regionalizadas (Matheron, 1971, 1968), com o propósito de descrever variáveis naturais caracterizadas por possuírem correlação espacial e/ou temporal, cuja principal aplicação foi direcionada para a estimativa de variáveis geológicas em depósitos minerais (Chilès \& Delfiner, 2012; Sinclair \& Blackwell, 2004).

As técnicas de estimativa geoestatísticas ( $p$ ex. Krigagem Ordinária) baseiam-se na continuidade espacial e/ou temporal da variável analisada, de tal maneira que os valores assumidos pela mesma não são totalmente aleatórios, mas possuem uma correlação espacial e/ou temporal, quantificada por meio da função variograma/semivariograma (Revuelta, 2018; Abzalov, 2016; Sinclair \& Blackwell, 2004; Isaaks \& Srivastava, 1989; Journel \& Huijbregts, 1978).

\subsubsection{Variograma}

O variograma $(2 \gamma(h))$ mensura o grau de variabilidade de uma variável em função da distância $(h)$ que separa pares de valores assumidos pela mesma, os quais estão atribuídos a um ponto no espaço ou no tempo (Rossi \& Deutsch, 2014). O grau de variabilidade para um dado valor de distância (Equação 3) é quantificado por meio de uma média das diferenças quadráticas entre dois valores experimentais $\left(z\left(x_{i}\right)\right.$ e $z\left(x_{i}+h\right)$ ) situados em quaisquer pontos $x_{i}$ e $x_{i}+h$ (Journel \& Huijbregts, 1978). Considerando-se um certo valor de distância são comparados $N(h)$ pares de pontos de dados experimentais separados pelo vetor $h$.

$2 \gamma(h)=\frac{1}{N(h)} \sum_{i=1}^{N(h)}\left[z\left(x_{i}\right)-z\left(x_{i}+h\right)\right]^{2}$

A metade da função variograma é denominada semivariograma $(\gamma(h))$, a qual é a ferramenta utilizada em aplicações práticas, de tal forma que o termo variograma, no desenvolver do trabalho, corresponderá ao que é de fato o semivariograma (Equação 4). 
$\gamma(h)=\frac{1}{2 N(h)} \sum_{i=1}^{N(h)}\left[z\left(x_{i}\right)-z\left(x_{i}+h\right)\right]^{2}$

Os valores de $\gamma(h)$ calculados para uma dada direção espacial podem ser plotados em função da distância, resultando em um gráfico constituído de pontos, cada um atribuído a um valor de $h$ (Figura 1), definido como "variograma experimental", o qual é ajustado por modelos matemáticos/teóricos para fins de estimativa (Abzalov, 2016; Sinclair \& Blackwell, 2004).

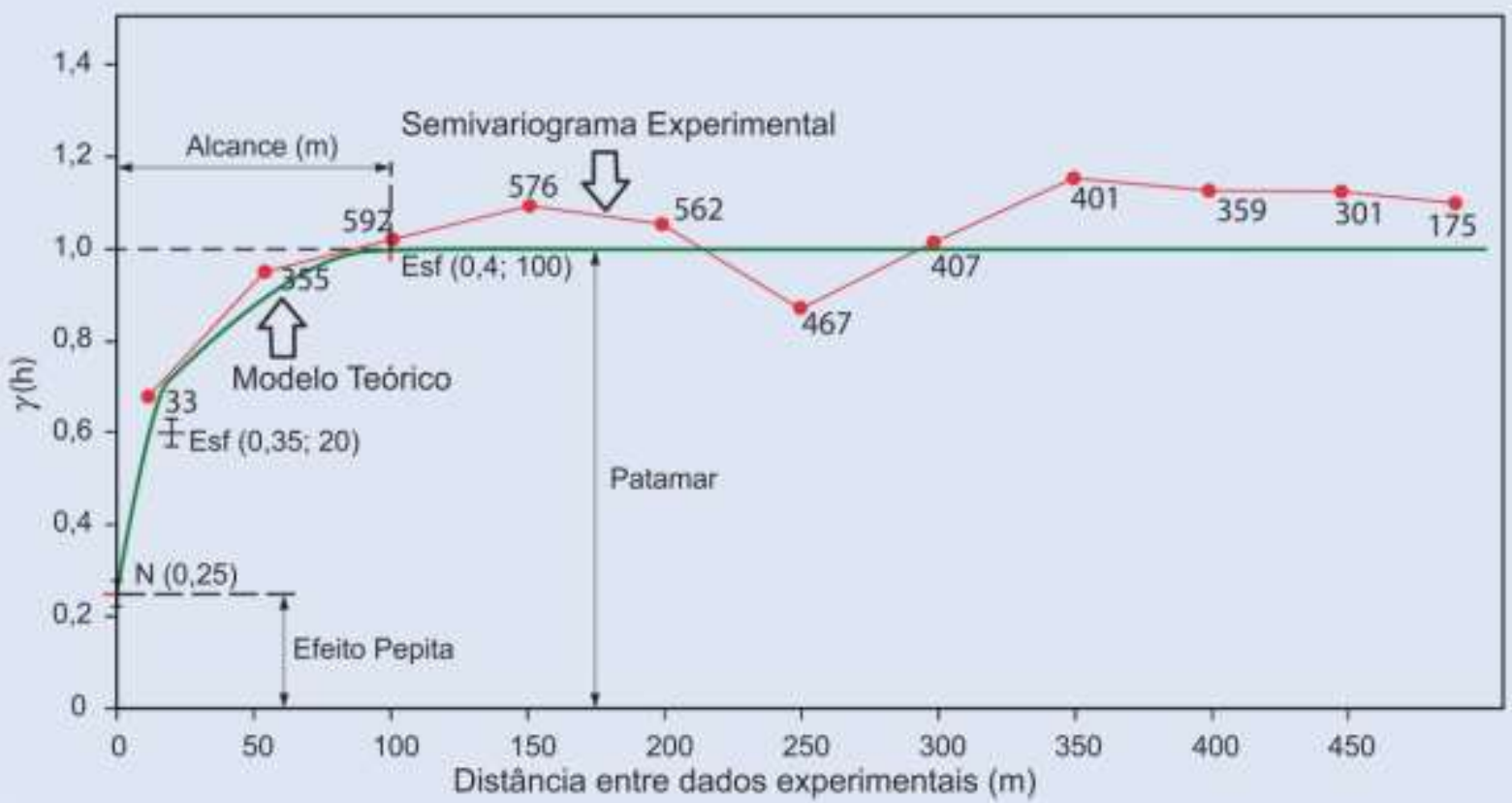

Figura 1: Variograma experimental (linha em vermelho), ajuste teórico (linha em verde) e parâmetros do variograma (Adaptado de Revuelta, 2018).

Um variograma típico (Figura 1) é caracterizado por três parâmetros: (i) Efeito Pepita, definido como uma descontinuidade na origem, cuja existência se deve principalmente à variabilidade associada a uma distância inferior ao menor espaçamento entre os pontos amostrais/experimentais (variabilidade de curta escala); (ii) Patamar, o qual é o valor de $\gamma(h)$ em que a correlação linear é zero, e corresponde normalmente à variância da variável sob análise; e (iii) Alcance, que é a distância a partir da qual não há correlação entre os pares de amostras comparados (Rossi \& Deutsch, 2014).

\subsubsection{Krigagem Ordinária}

A Krigagem Ordinária é um método de estimativa/interpolação geoestatístico baseado na combinação linear de dados experimentais $\left(Z\left(x_{i}\right)\right)$ vizinhos (Equação 5$)$, cujo propósito é obter uma estimativa $\left(Z_{K O}^{*}\left(x_{0}\right)\right)$ em um ponto/bloco localizado em $x_{0}$ com mínima variância/erro de krigagem, de tal forma que os ponderadores $\left(\lambda_{i}\right)$ obtidos pelo sistema (Equação 6) são ótimos, o que resulta em valores ótimos de estimativa, sob a restrição de não enviesamento, a qual delimita que a média do erro de estimativa deve ser nula (Yamamoto \& Landim, 2013; Journel \& Huijbregts, 1978). 


$$
Z_{K O}^{*}\left(x_{0}\right)=\sum_{i=1}^{n} \lambda_{i} Z\left(x_{i}\right)
$$

$$
\left[\begin{array}{c}
\lambda_{1} \\
\lambda_{2} \\
\vdots \\
\lambda_{n} \\
-\mu
\end{array}\right]=\left[\begin{array}{ccccc}
\gamma\left(x_{1}-x_{1}\right) & \gamma\left(x_{1}-x_{2}\right) & \ldots & \gamma\left(x_{1}-x_{n}\right) & 1 \\
\gamma\left(x_{2}-x_{1}\right) & \gamma\left(x_{2}-x_{2}\right) & \ldots & \gamma\left(x_{2}-x_{n}\right) & 1 \\
\vdots & \vdots & \ldots & \vdots & \vdots \\
\gamma\left(x_{n}-x_{1}\right) & \gamma\left(x_{n}-x_{2}\right) & \cdots & \gamma\left(x_{n}-x_{n}\right) & 1 \\
1 & 1 & \ldots & 1 & 0
\end{array}\right]^{-1}\left[\begin{array}{c}
\gamma\left(x_{0}-x_{1}\right) \\
\gamma\left(x_{0}-x_{2}\right) \\
\vdots \\
\gamma\left(x_{0}-x_{n}\right) \\
1
\end{array}\right]
$$

Na Equação (6), os termos $\gamma\left(x_{i}-x_{j}\right)$ correspondem ao variograma entre os pares $x_{i}$ e $x_{j}$, distanciados entre si por um vetor $h$.

A variância de krigagem, neste método de estimativa, é obtida por meio da Equação (7), a qual relaciona: (i) os ponderadores ótimos obtidos por meio do sistema de krigagem (Equação 6); (ii) os valores de variograma $\left(\gamma\left(x_{0}-x_{i}\right)\right)$ entre o ponto a estimar $\left(x_{0}\right)$ e os dados experimentais vizinhos $\left(x_{i}\right)$; e (iii) o parâmetro de Lagrange $(\mu)$, derivado da lagrangeana (Journel \& Huijbregts, 1978).

$\sigma_{K O}^{2}=\sum_{i=1}^{n} \lambda_{i} \gamma\left(x_{0}-x_{i}\right)+\mu$

\section{METODOLOGIA}

O banco de dados numérico utilizado para a realização do mapeamento de falhas estruturais foi baseado em valores de métrica de dano em uma placa de alumínio de $30 \mathrm{~cm} \times 30$ $\mathrm{cm}$, onde foi estipulada uma malha regular com 24 pontos. Considerando-se a localização do dano (Figura 2) e a magnitude do mesmo, foram definidos quatro cenários, cujas características estão expostas na Tabela 1. 

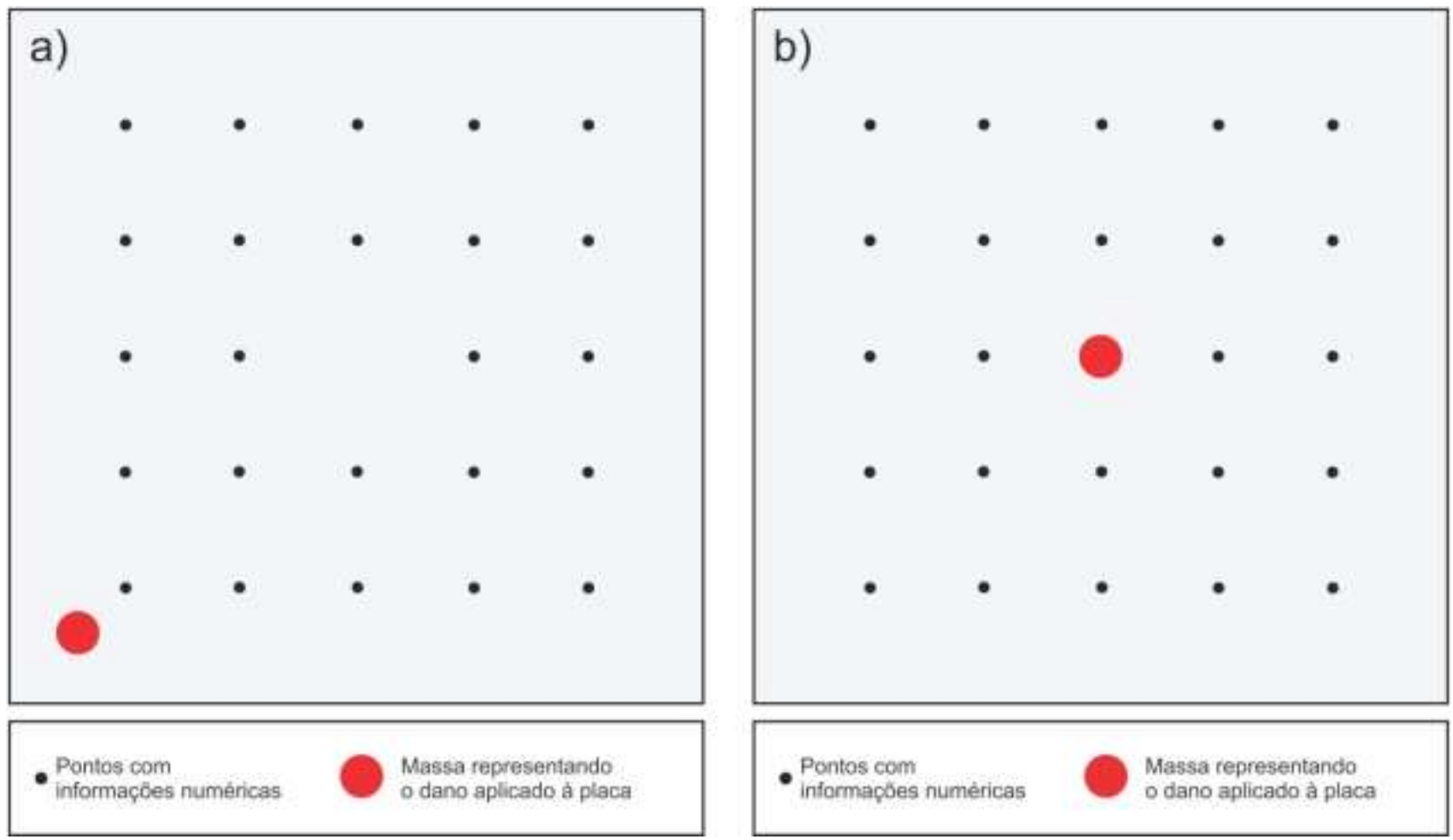

Figura 2: Disposição dos pontos nos cenários - a) com a massa na parte inferior esquerda; b) com a massa no centro da placa

Tabela 1: Características dos cenários considerados para o mapeamento das métricas de dano.

\begin{tabular}{c|c|c|c}
\hline Cenário & Variável & Massa $(\mathrm{g})$ & Posição na placa \\
\hline C1 & D1 & 7,0 & Inferior esquerda \\
C2 & D2 & 14,0 & Inferior esquerda \\
C3 & D3 & 7,0 & Centro \\
C4 & D4 & 14,0 & Centro \\
\hline
\end{tabular}

A partir das informações de métrica de dano, foi realizada a análise estatística - por meio de histogramas - da variável em cada cenário considerado ( $C 1, C 2, C 3$ e C4), extraindo-se os indicadores de tendência central e de dispersão, com o propósito de: (i) observar o comportamento estatístico da métrica de dano em cada situação; e (ii) obter, para cada cenário, a variância da variável, a qual normalmente corresponde à variância a priori (patamar total) dos respectivos variogramas.

Na sequência, para cada cenário, foram calculados variogramas experimentais com o propósito de identificar a ocorrência ou não de direções preferenciais de continuidade espacial (anisotropia) da variável. De posse de tal análise, foram efetuados, em cada cenário, os ajustes variográficos (ajuste teórico) de dois variogramas experimentais, perpendiculares entre si, os quais definem a continuidade espacial da métrica de dano em cada situação.

A estimativa dos valores de métrica de dano em cada cenário foi efetuada por meio de Krigagem Ordinária (KO) a partir dos respectivos parâmetros dos ajustes variográficos e dos valores numéricos da métrica de dano. A KO foi definida como o estimador pelo fato de ser um método 
mais apropriado para descrever variações locais em estimativas geoestatísticas, não necessitando, também, da média populacional da variável, como no caso da Krigagem Simples (KS) para a execução das estimativas.

Em cada situação considerada, os valores foram estimados em uma grade cartesiana (grid) com blocos quadrados de $1,5 \mathrm{~cm}$ de lado, cuja dimensão foi definida de tal forma com o propósito de mapear de maneira local as variações da métrica de dano na placa.

\section{RESULTADOS E DISCUSSÕES}

A análise estatística das variáveis D1, D2, D3 e D4 por meio de histogramas (Figura 3) mostraram que os mesmos possuem assimetria positiva, com uma frequência maior de valores mais baixos. $O$ formato da distribuição, a partir da quantidade de valores utilizada não pôde ser determinado, necessitando-se de uma quantidade maior de amostras para se avaliar o mesmo estatisticamente, por meio de testes de hipóteses.

Os resultados da análise estatística mostram também que as variáveis D2 e D4 possuem, de maneira geral, valores maiores de métrica de dano, o que se justifica pelo fato de que estas encontram-se associadas a uma massa/dano maior em relação às variáveis D1 e D3.
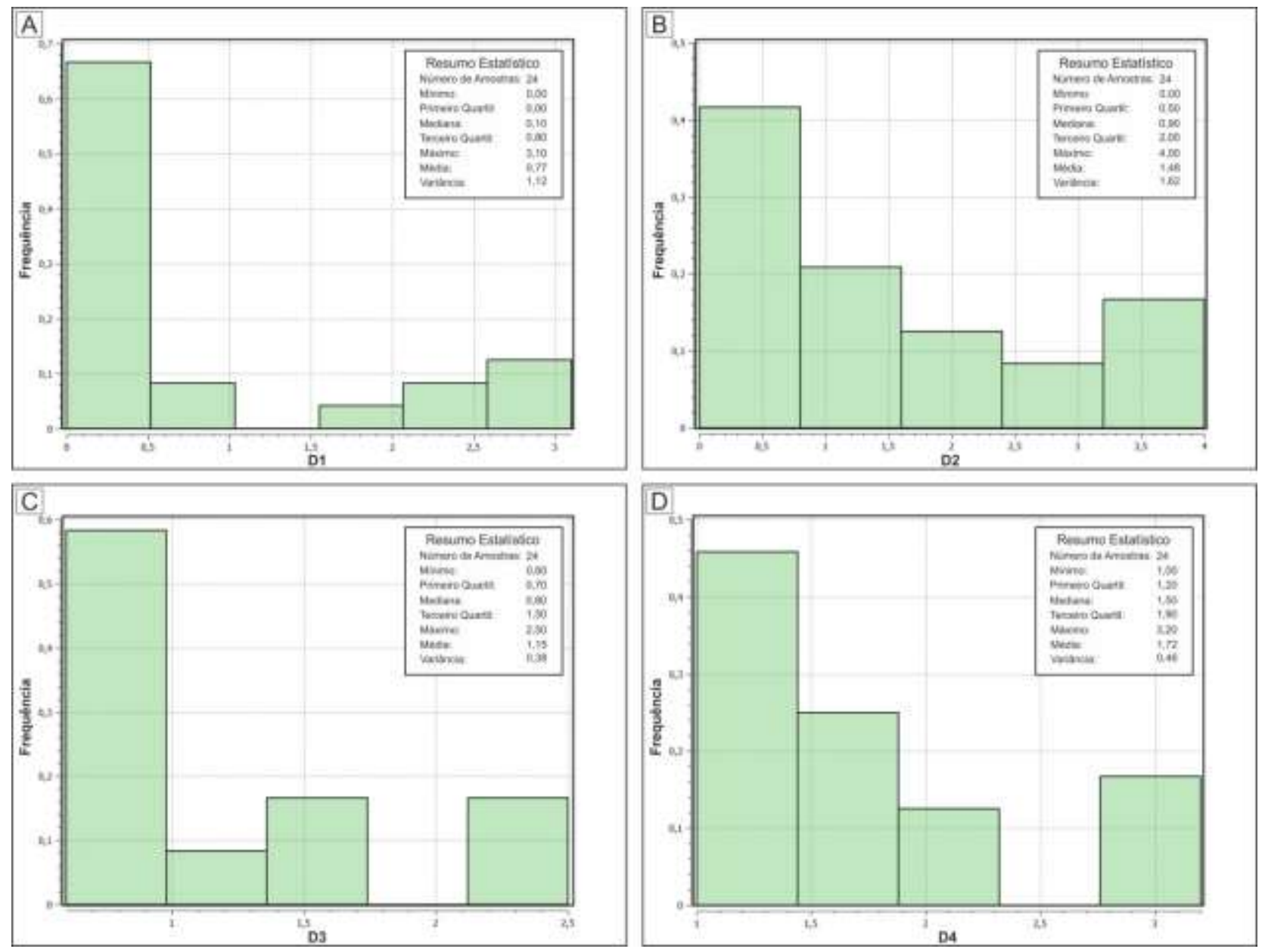

Figura 3: Histogramas das variáveis - a) D1; b) D2; c) D3; d) D4.

O cálculo de variogramas em várias direções mostraram que as direções preferenciais de continuidade estavam nas direções Norte-Sul (azimute de $0^{\circ}$ ) e Leste-Oeste (azimute de $90^{\circ}$ ), onde 
a direção Leste-Oeste correspondeu à direção de maior continuidade espacial dentre as variáveis avaliadas, com exceção da variável D4, na qual a direção mais contínua foi a direção Norte-Sul. A Figura 4 mostra os variogramas calculados (pontos em vermelho) e os respectivos ajustes teóricos (linha contínua em preto), e a Tabela 2, os parâmetros dos ajustes variográficos.

Tabela 2: Parâmetros do ajuste variográfico de D1, D2, D3 e D4.

\begin{tabular}{c|c|c|c|c|c}
\hline \multirow{2}{*}{ Variável } & \multirow{2}{*}{ Orientação } & \multirow{2}{*}{ Efeito Pepita } & \multicolumn{3}{|c}{ Estrutura 1 } \\
\cline { 4 - 6 } & & & Modelo & Contribuição & Alcance (cm) \\
\cline { 4 - 6 } & & 0,000 & Gaussiano & 1,123 & 14,0 \\
\multirow{2}{*}{ D1 } & Horizontal 0 & Horizontal 90 & & & \\
& Horizontal 0 & 0,000 & Gaussiano & 1,123 & 15,0 \\
\hline \multirow{2}{*}{ D2 } & Horizontal 90 & 0,000 & Gaussiano & 1,624 & 14,1 \\
& Horizontal 0 & 0,000 & Gaussiano & 1,624 & 20,0 \\
\hline \multirow{2}{*}{ D3 } & Horizontal 90 & 0,000 & Esférico & 0,382 & 10,0 \\
& Horizontal 0 & 0,000 & Esférico & 0,382 & 10,5 \\
\hline \multirow{2}{*}{ D4 } & Horizontal 90 & 0,000 & Esférico & 0,461 & 12,0 \\
& & & & 7,8 \\
\hline
\end{tabular}



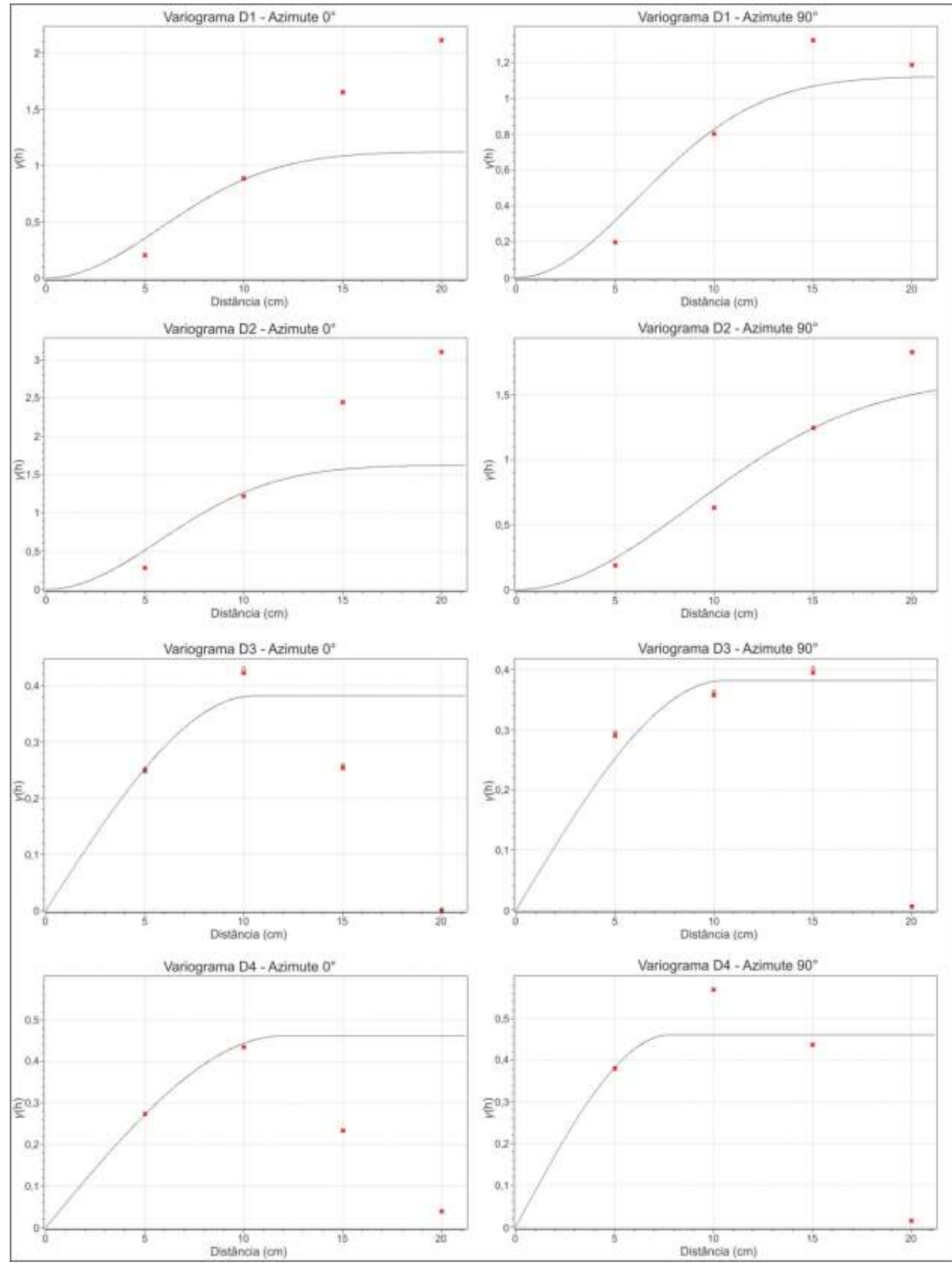

Figura 4: Variogramas experimentais e ajustes teóricos para cada variável, para cada direção preferencial. 
Os variogramas calculados não apresentaram uma quantidade razoável de pontos para que o ajuste teórico fosse executado com precisão, de tal maneira que uma quantidade maior de informações seria necessária para a determinação mais assertiva dos parâmetros do modelo, particularmente o Efeito Pepita (neste caso, nulo em todas as variáveis) e o tipo de modelo teórico.

Quanto ao tipo de modelo, as variáveis D1 e D2 apresentaram um melhor ajuste por meio do modelo Gaussiano, o qual demonstra comparativamente um fenômeno mais contínuo em relação ao modelo esférico, utilizado para o ajuste das variáveis D3 e D4. Portanto, através dessas informações, conclui-se que os valores de métrica de dano em D1 e D2 são mais homogêneos espacialmente em relação aos valores encontrados em D3 e D4. Entretanto, uma análise com uma quantidade maior de informações se faz necessária para a determinação mais precisa do modelo a ser utilizado no ajuste variográfico.

Avaliando-se a razão de anisotropia das variáveis, calculada pela relação entre os respectivos maior e menor alcances variográficos (Tabela 2), observou-se que os menores valores de tal indicador foram atribuídos às variáveis D1 $(1,07)$ e D3 $(1,05)$, nas quais a massa utilizada para representar o dano estrutural é menor em relação às demais. A aplicação de uma massa maior resultou em razões de anisotropia mais elevadas, conforme pode ser observado nas variáveis D2 e D4, cujos valores deste indicador foram 1,42 e 1,54, respectivamente, o que pode ser um indicativo de existência de direção preferencial de propagação do dano.

Os resultados das estimativas, de acordo com o que mostram os mapas da Figura 5, reproduziram os maiores valores de falha nos locais em que foram posicionadas as massas para denotar o dano na placa. Assim, nos mapas das variáveis D1 e D2, os maiores valores de dano estiveram localizados no canto inferior e esquerdo da placa, enquanto que nos mapas das variáveis D3 e D4 os maiores valores de dano estiveram posicionados no centro da placa. Além disso, é possível observar uma maior área relacionada ao dano estrutural nos mapas das variáveis D2 e D4, as quais estão vinculadas a uma massa maior, refletindo, portanto, em um dano estrutural maior.

Nos mapas das variáveis D2 e D4 (Figura 5), é possível observar a existência de uma direção preferencial de continuidade para os valores de métrica de dano, em que esta se propaga de maneira mais contínua na estrutura, indicando, portanto, a existência de um dano que se propaga mais nestas posições. Neste contexto, pode-se observar que para a variável D2, o dano se propagaria preferencialmente na direção Leste-Oeste, enquanto que para a variável D4, na direção Norte-Sul. 

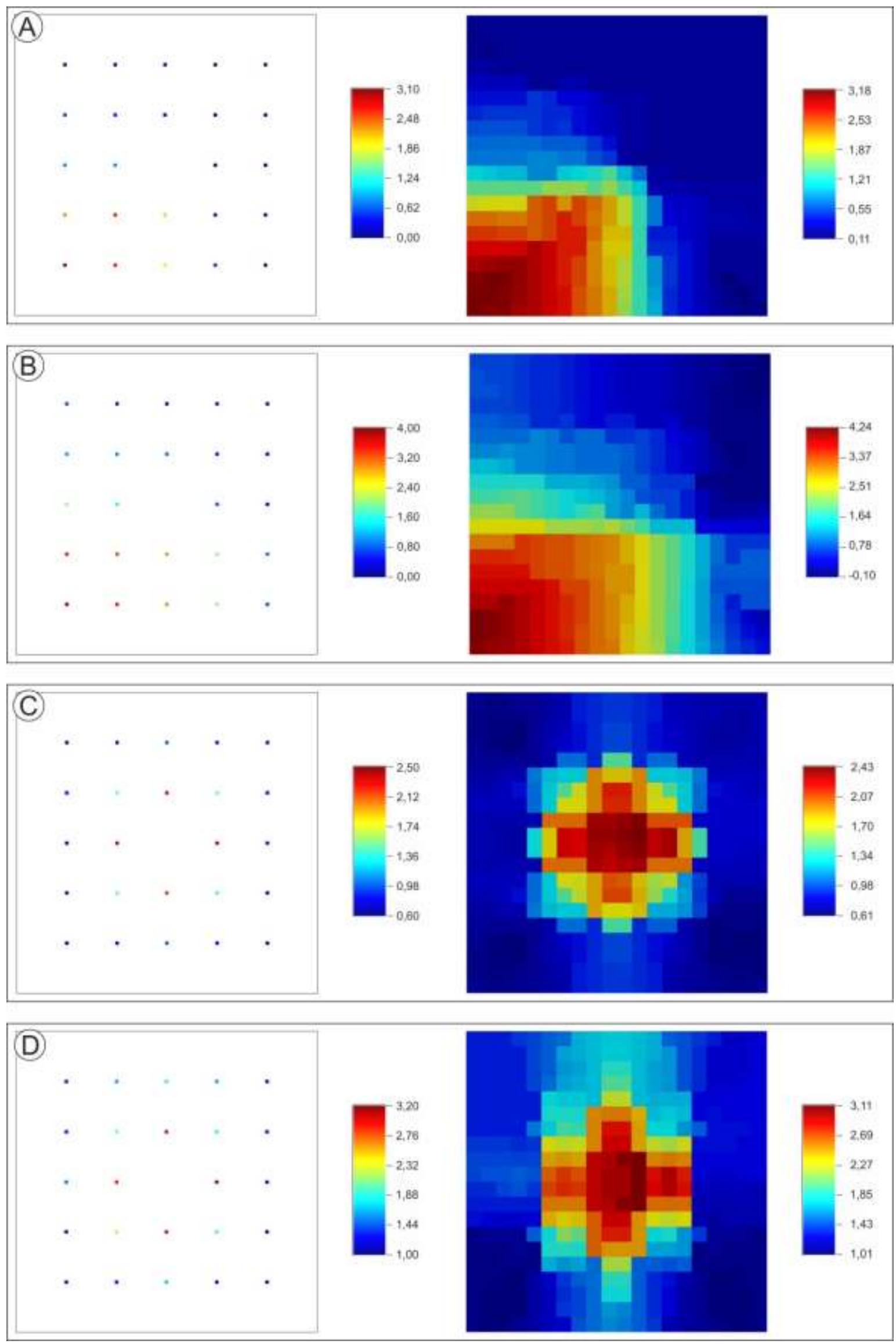

Figura 5: Mapas dos resultados das estimativas para as variáveis - a) D1; b) D2; c) D3; d) D4. 
As distribuições estatísticas reais (amostras/dados numéricos) e estimadas, comparadas entre si nos gráficos Q-Q (Figura 6), mostraram que as respectivas médias reais e estimadas se mostraram compatíveis, de tal maneira que houve aproximadamente uma reprodução das médias reais. Entretanto, em todas as variáveis houve desvios acentuados para valores amostrais acima dos respectivos terceiros quartis (áreas em verde na Figura 6), o que pode ser uma consequência do efeito suavizador do método de estimativa e/ou dos ajustes variográficos, os quais podem ser aprimorados pela adição de novas informações.
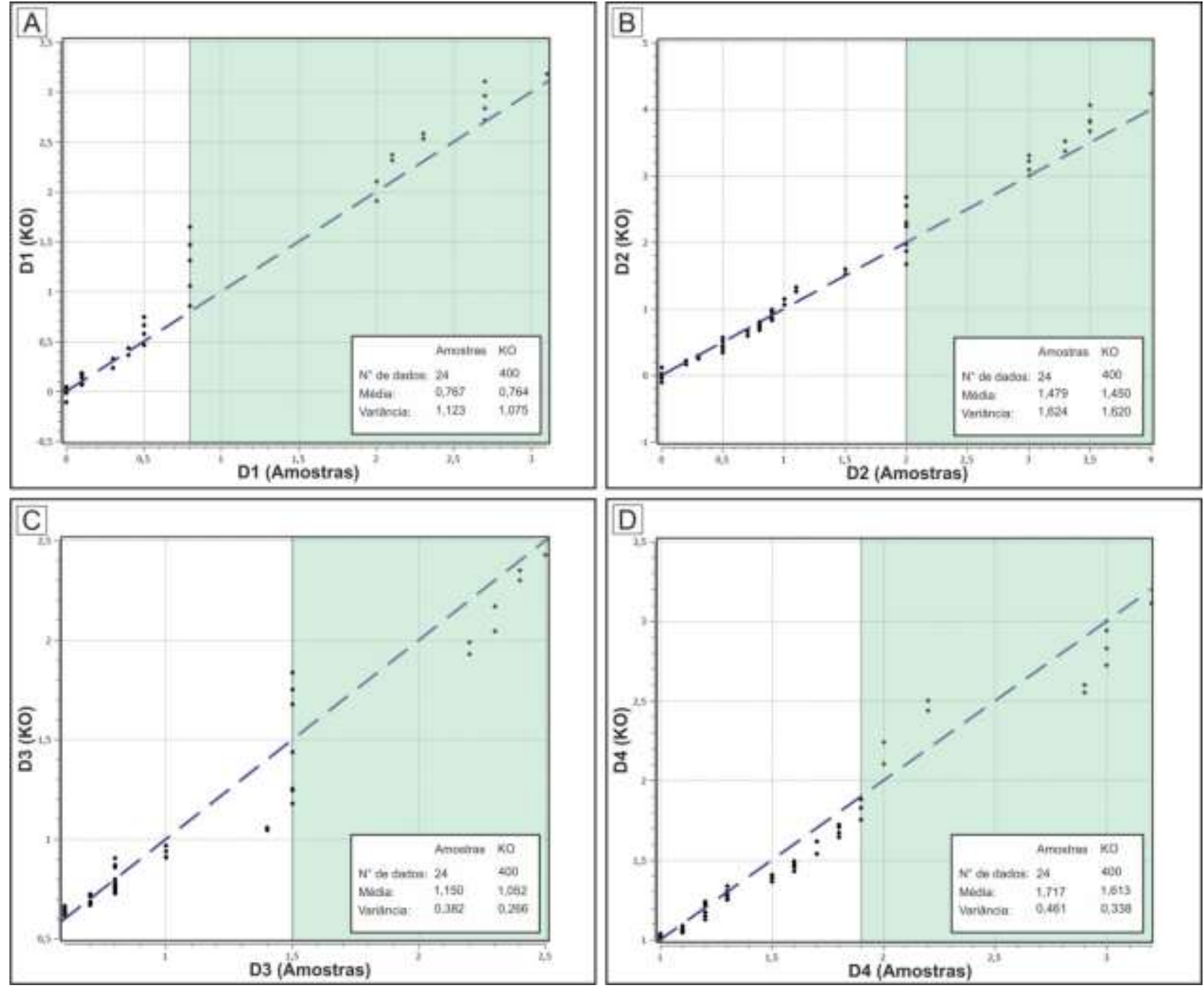

Figura 6: Gráficos Q-Q das distribuições estatísticas reais (amostras) e estimadas das variáveis - a) D1; b) D2; c) D3; d) D4.

Os mapas da variância de Krigagem Ordinária (Figura 7) mostram que os menores valores de variância estão associados às áreas em que há valores numéricos, indicando erros de estimativa menores nestas áreas. À medida em que há um distanciamento dos valores numéricos, há um aumento do erro de estimativa (variância de krigagem), cujos maiores valores estiveram situados nas bordas da placa em todos os casos analisados.

Os resultados evidenciam que a variância de krigagem pode auxiliar na delimitação de áreas com deficiência de sensores (PZTs), as quais possuiriam valores elevados de variância. A partir de tal 
informação seria possível estabelecer malhas mais eficientes, no sentido de coletar adequadamente as informações da estrutura.
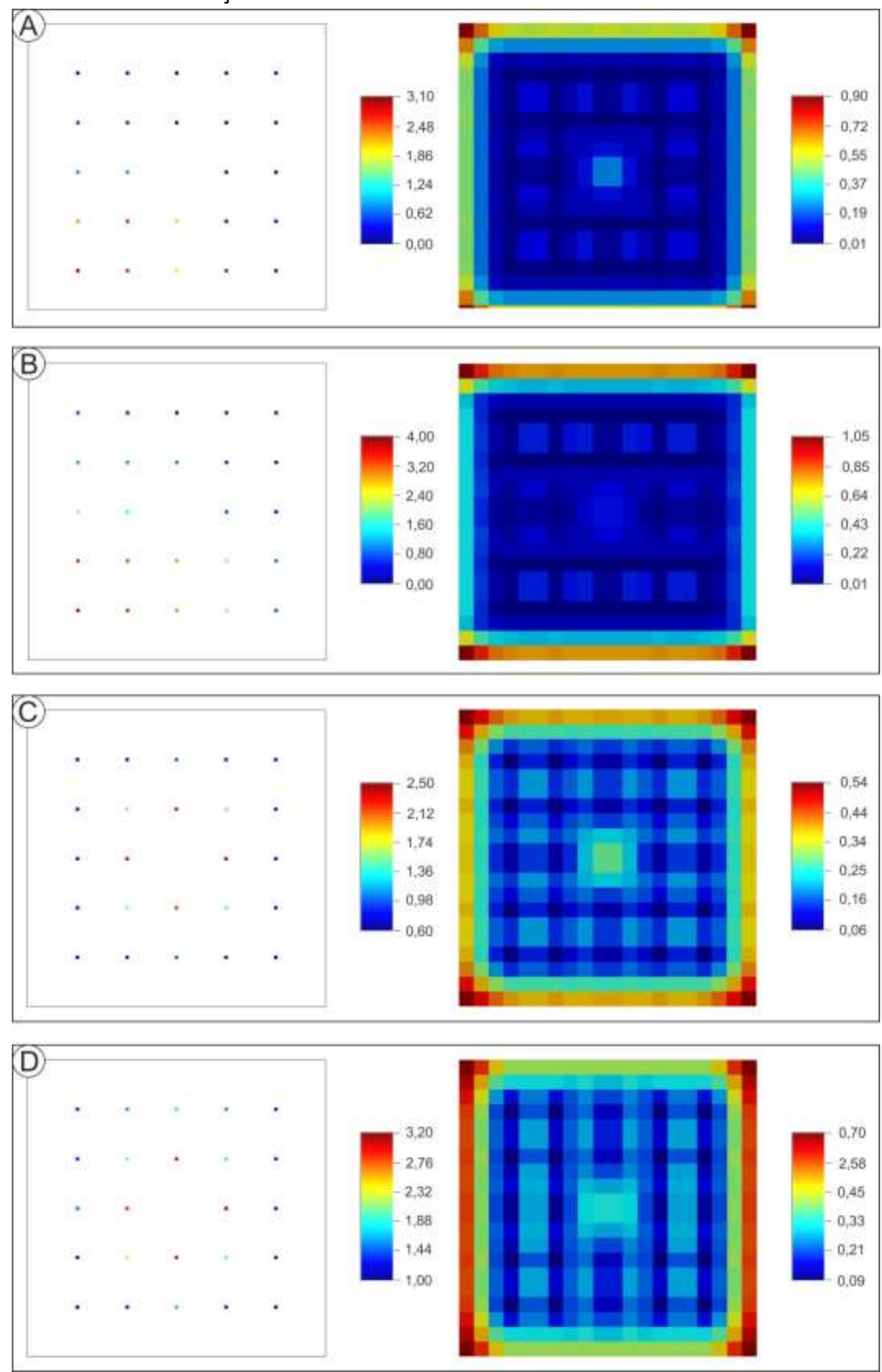

Figura 7: Mapas da variância de Krigagem Ordinária para as variáveis - a) D1; b) D2; c) D3; d) D4 


\section{CONCLUSÕES}

A utilização de Krigagem Ordinária para interpolar valores de métrica de dano estrutural baseados em impedância eletromecânica (E/M) se mostrou promissora. Apesar do efeito de suavização das estimativas, característica do método, foi possível mapear o dano estrutural em quatro situações distintas, cujos resultados se mostraram visualmente compatíveis com os dados numéricos.

A comparação entre os indicadores estatísticos dos dados numéricos e das respectivas estimativas mostraram que as médias foram aproximadamente reproduzidas, cujo maior desvio neste indicador foi atribuído à variável D3 (8,52\%), seguido de D4 (6,06\%), D2 (1,96\%) e D1 (0,39\%). Tal comportamento é previsível, uma vez que o estimador utilizado é capaz de reproduzir melhor valores próximos da média, enquanto que valores dos extremos da distribuição apresentam maiores desvios na estimativa (efeito de suavização).

Os desvios entre as distribuições real e estimadas (gráficos Q-Q) podem ser reduzidos a partir do acréscimo de informações, o que possibilitaria também a determinação de variogramas experimentais mais robustos, o que aumentaria a qualidade das estimativas.

A variância de krigagem, a qual denota o erro cometido na estimativa, pode ser utilizada com o propósito de estabelecer malhas de sensores que permitam um mapeamento mais eficiente da estrutura, uma vez que delimita as áreas com os maiores erros de estimativa, as quais correspondem àquelas com deficiência de sensores.

A partir da análise dos resultados, conclui-se que os comparativos entre os indicadores estatísticos mostram que o método de se usar dados numéricos baseados em métricas de dano tem boa aplicabilidade na detecção de falhas na estrutura. Tal aplicação pode ser feita, em trabalhos futuros, utilizando dados reais (coletados) de uma estrutura, utilizando a técnica de SHM baseada em impedância eletromecânica, a fim de comprovar a utilização do método de Krigagem na detecção de falhas e suas intensidades.

\section{REFERÊNCIAS}

ABZALOV, M. (2016). Applied Mining Geology. Cham, Suíça: Springer International Publishing AG. https://doi.org/10.1007/978-3-319-39264-6

BAO, Y., CHEN, Z., WEI, S., XU, Y., TANG, Z., \& LI, H. (2019). The State of the Art of Data Science and Engineering in Structural Health Monitoring. Engineering, v. 5, p. 234-242. https://doi.org/10.1016/i.eng.2018.11.027

BAPTISTA, F. G. (2010). Uma contribuição aos sistemas de Monitoramento de Integridade Estrutural baseados na Impedância Eletromecânica (Tese de Doutorado). Ilha Solteira, Brasil: UNESP. Recuperado de https://repositorio.unesp.br/handle/11449/100277

BHALLA, S., \& SOH, C. K. (2012). Electro-Mechanical Impedance Technique. In C. K. Soh et al. (Eds.), Smart Materials in Structural Health Monitoring, Control and Biomechanics (1 ed., pp. 17-51). Heidelberg, Alemanha: Springer-Verlag. https://doi.org/10.1007/978-3-642-24463-6 
BRAY, D. E., \& MCBRIDE, D. (1992). Nondestructive testing techniques. Nova lorque, NY: John Wiley \& Sons Ltd.

CHILÈS, J. P., \& DELFINER, P. (2012). Geostatistics - Modeling Spatial Uncertainty (2 ed.). Hoboken, NJ: John Wiley \& Sons. https://doi.org/10.1002/9781118136188

DHILLON, B. S. (2008). Mining Equipment Reliability, Maintainability, and Safety. Londres, Inglaterra: Springer-Verlag London.

FARRAR, C. R., LIEVEN, N. A., \& BEMENT, M. (2005). An Introduction to Prognosis. In D. J. Inman et al. (Eds.), Damage Prognosis: For Aerospace, Civil and Mechanical Systems (1 ed., pp. 112). Chichester, Inglaterra: John Wiley \& Sons Ltd.

GIURGIUTIU, V. (2014). Structutal Health Monitoring with Piezoelectric Wafer Active Sensors (2 ed.). Waltham, MA: Academic Press. https://doi.org/10.1016/C2013-0-00155-7

GIURGIUTIU, V., \& ROGERS, C. A. (1998). Recent advancements in the electromechanical (E/M) impedance method for structural health monitoring and NDE. In: Annual International Symposium on Smart Structures and Materials, 5, 1998. San Diego, CA. Proceedings SPIE, v. 3329, p. 536-547. https://doi.org/10.1117/12.316923

GOPALAKRISHNAN, S., RUZZENE, M., \& HANAGUD, S. (2011). Computational Techniques for Structural Health Monitoring (1 ed.). Londres, Inglaterra: Springer-Verlag London Limited. https://doi.org/10.1007/978-0-85729-284-1

ISAAKS, E. H., \& SRIVASTAVA, R. M. (1989). An Introduction to Applied Geostatistics. New York, NY: Oxford University Press.

Journel, A. G., \& HUIJBREGTS, Ch. J. (1978). Mining Geostatistics. San Diego, CA: Academic Press Limited.

LIANG, C., SUN, F. P., \& ROGERS, C. A. (1994). Coupled electro-mechanical analysis of adaptive material systems - Determination of the actuator power consumption and system energy transfer. Journal of Intelligent Material Systems and Structures, v. 5, n. 1, p. 12-20. https://doi.org/10.1177/1045389X9400500102

MARUO, I. I. C., GIACHERO, G. F., STEFFEN JUNIOR, V., \& FINZI NETO, R. M. (2015). Electromechanical Impedance - Based Structural Health Monitoring Instrumentation System Applied to Aircraft Structures and Employing a Multiplexed Sensor Array. J. Aerosp. Technol. Manag., v. 7, n. 3, p. 294-306. https://doi.org/10.5028/jatm.v7i3.447

MATHERON, G. (1963). Principles of geostatistics. Economic Geology, v. 58, n. 8, p. 1246-1266. https://doi.org/10.2113/gsecongeo.58.8.1246

MATHERON, G. (1971). The theory of regionalized variables and its applications. Fontainebleau, França: École national supérieure des mines. 
MOURA JUNIOR, J. R. V. (2008). Uma contribuição aos sistemas de monitoramento de integridade estrutural aplicada a estruturas aeronáuticas e espaciais (Tese de Doutorado). Uberlândia, Brasil: UFU. Recuperado de https://repositorio.ufu.br/handle/123456789/14674

REVUELTA, M. B. (2018). Mineral Resources - From Exploration to Sustainability Assessment. Cham, Suíça: Springer International AG. https://doi.org/10.1007/978-3-31958760-8

ROSSI, M. E., \& DEUTSCH, C. V. (2014). Mineral Resource Estimation. Dordrecht, Holanda: Springer Science+Business Media. https://doi.org/10.1007/978-1-4020-5717-5

SILVA, R. (2017). Monitoramento de integridade estrutural utilizando a técnica de impedância eletromecânica aplicada em estruturas de concreto (Tese de Doutorado). Uberlândia, Brasil: Universidade Federal de Uberlândia.

SINCLAIR, A. J., \& BLACKWELL, G. H. (2004). Applied Mineral Inventory Estimation. Cambridge, Reino Unido: Cambridge University Press. https://doi.org/10.1017/CBO9780511545993

YAMAMOTO, J. K., \& LANDIM, P. M. B. (2013). Geoestatística: Conceitos e Aplicações (1 ed.). São Paulo, Brasil: Oficina de Textos.

YAN, R., CHEN, X., \& MUKHOPADHYAY, S. C. (2017). Advanced Signal Processing for Structural Health Monitoring. In R. Yan et al. (Eds.), Structural Health Monitoring: An Advanced Signal Processing Perspective (1 ed., pp. 1-11). Cham, Suíça: Springer International AG. https://doi.org/10.1007/978-3-319-56126-4

ZAGRAI, A. N., \& GIURGIUTIU, V. (2009). Electromechanical Impedance Modeling. In C. Boller et al. (Eds.), Encyclopedia of Structural Health Monitoring (1 ed., pp. 71-89). New York, NY: John Wiley \& Sons. https://doi.org/10.1002/9780470061626 